Check for updates

Cite this: RSC Adv., 2017, 7, 25861

\title{
Self-formed nanogap junctions for electronic detection and characterization of molecules and quantum dots $\dagger$
}

\author{
Amir Ziv, (D) a Avra Tzaguy, ${ }^{\text {b }}$ Ori Hazut, ${ }^{\text {b }}$ Shira Yochelis, ${ }^{a}$ Roie Yerushalmi (DD *b \\ and Yossi Paltiel ${ }^{* a}$
}

Fabrication of self-forming nanojunction devices is demonstrated using positioning of nanofloret-like building blocks that serve as self-assembled electrodes. A main feature of the device is a self-formed nanogap bridging between the nanofloret (NF) hybrid nanostructures (HNS) and a macroscopic counterelectrode. When nanostructures are introduced to the device they provide facile bridging across the nanostructure. This strategy is used to demonstrate electronic measurements across molecules and nanoparticles. Connecting the NF nanojunction to the micro-, and macro-scales is achieved by applying standard, robust, optical lithography. In addition, the devices are operable at ambient conditions and in solvent environments, where introducing molecules to the device results in a prominent change in the conductance characteristics. Furthermore, introduction of quantum dots results in the mapping of their band structure at ambient conditions. Our results provide a proof-of-concept of large scale self-forming nanogap device platform realized using simple fabrication tools. Such a technology can be used for molecular detectors, as a potential building block for molecular electronics, or as a platform for fundamental research.

Received 24th April 2017

Accepted 5th May 2017

DOI: $10.1039 / \mathrm{c} 7 \mathrm{ra0} 4600 \mathrm{f}$

rsc.li/rsc-advances

\section{Introduction}

Coupling standard electronic circuits with large size contacts to the nanoscale molecular-level and nanoparticles is an attractive concept with intense research and technological activity. ${ }^{1-5}$ Central to this concept is the transport of charge carriers through a single molecule, or a small ensemble of molecules. Transport properties in molecules exploit the richness, versatility, repeatability and functionality that are the hallmarks of molecular components in the context of electronic devices. ${ }^{1}$ Current synthesis methods offer a large variety of molecules and nanoscale building blocks with high purity, tunable electronic structure, connectivity and functionalities, together with uniform size and composition. This makes them attractive candidates for integration as electronic components. However, despite these advances, substantial obstacles are still present when coupling molecular and nanoscale building blocks to external electrodes, which hamper the exploitation of their huge potential for large scale electronic components. These

${ }^{a}$ Department of Applied Physics, The Hebrew University of Jerusalem, 9190401 Israel. E-mail: paltiel@mail.huji.ac.il

${ }^{b}$ Institute of Chemistry, The Center for Nanoscience and Nanotechnology, The Hebrew University of Jerusalem, Edmond J. Safra Campus, Givat Ram, Jerusalem, 9190401, Israel. E-mail: roie.yerushalmi@mail.huji.ac.il

$\dagger$ Electronic supplementary information (ESI) available. See DOI: $10.1039 / \mathrm{c} 7 \mathrm{ra} 04600 \mathrm{f}$ difficulties are both technical and fundamental in nature. One technical challenge is the fabrication of contacts to the element under study, such as a molecule, or a quantum dot, without causing damage or altering its electronic properties. Two of the key fundamental issues are (i) keeping the connection stable for long time periods, and (ii) controlling the chemical or physical bonding between the element under study and the electrodes. ${ }^{6}$ A promising approach for overcoming some of these obstacles involves nanogap architectures with good conductivity to the respective element under study, which is also commonly termed registry. Fabricating nanogap devices with specific dimensions is not simple and usually requires complicated technology, making the fabrication yield of two-terminal connectivity nanogap junctions quite low. Indeed, significant efforts have been dedicated toward the realization and study of nanogap architectures for diverse applications including molecular rectifiers, switching and electro-chemical sensors. ${ }^{7-9}$ These efforts yielded great advances in the study of the transport properties of molecular junctions in two-terminal devices, serving as a powerful platform for research at the singlemolecule level. Similar studies were conducted using STM/ AFM-based junctions, ${ }^{10}$ mechanically-controlled break junctions ${ }^{11}$ and electromigration-controlled gaps. ${ }^{12}$ While all these platforms yielded fundamental insights and research tools for charge and energy transport for single molecules ${ }^{13}$ the fabrication methods are usually complex and require advanced e- 
beam lithography, high-vacuum conditions, or complicated feedback mechanisms for junction formation.

These methods inhibit the realization and integration of these classes of devices. Fabricating small and compact junctions usually requires complex, multi-step processes which are not easily reproducible or scalable, and are not compatible with standard clean room methods. ${ }^{\mathbf{1 2 , 1 4}}$ On the other-hand, simple methods for fabricating nanogaps, usually have low control over gap size ${ }^{15}$ or are limited in scaling the junction cross section. ${ }^{16,17}$ Therefore, it is highly desired to develop nanogap device fabrication schemes that would be compatible with standard silicon device technologies. Such hybrid integrated circuits have the potential to be operated, tested, and to function under ambient conditions and may open the way for utilization of such devices in numerous applications.

Here, we present a fabrication method for a self-formed nanojunction based on nanofloret (NF) hybrid nanostructures (HNS) using SiGe nanowires (NWs) grown at selective locations. The fabrication process is robust, using simple lithographic methods (top-down) for the micron-scale parts of the device, and bottom-up methods for the nanoscale elements. The nanogap is self-formed, created by wet processing at ambient conditions, whereas the gap size is adjustable according to the trapped nanostructure. High geometrical aspect ratio SiGe NWs are used to form the nanofloret HNS where a metallic nanoshell cap that function as a contact is deposited selectively at the NW tip. ${ }^{18}$ The length of the NWs can be tuned between 1-10 microns, enabling to bridge the gap between two distant optical lithography fabricated contacts. The active junction is formed between the edge of the NF where the metallic cap is deposited by wet process and the counter electrode with a very small contact area owing to the geometry of the NF HNS. $I-V$ transport measurements performed after the introduction of molecules and quantum dots (QD) into the system reveal that they control and change the junction conductance, thus establishing a simple molecular detector and provides information on their electronic structures. The resulting platform is a first step towards achieving a simple and robust method to study and control of molecular- and nanostructure-based electronic devices.

\section{Results and discussion}

Typical fabrication of self-forming nanogap junction device includes the following process steps, depicted in Fig. 1a: (I) gold nanoparticles are selectively adsorbed at the edge of one of the two prefabricated Ti contacts separated by a micrometric gap. (II) The sample is inserted into a Chemical Vapor Deposition (CVD) system, where SiGe nanowires are grown where the gold nanoparticles act as growth catalysts, similar to related works. ${ }^{19-22}$

The length of the NWs is controlled by the CVD growth parameters and deposition time and the density and position of NWs seeds is controlled by the selective adsorption of nanoparticles. (III) Above several $\mu \mathrm{m}$ in length, the NWs do not stand vertically but rather fall and lay horizontally, where the ones at the edge of the contact tends to fall outwards, thus bridging the gap between the contacts. (IV) NF HNS are synthesized as described previously, ${ }^{\mathbf{1 8}}$ the wetting and drying of the samples (a)
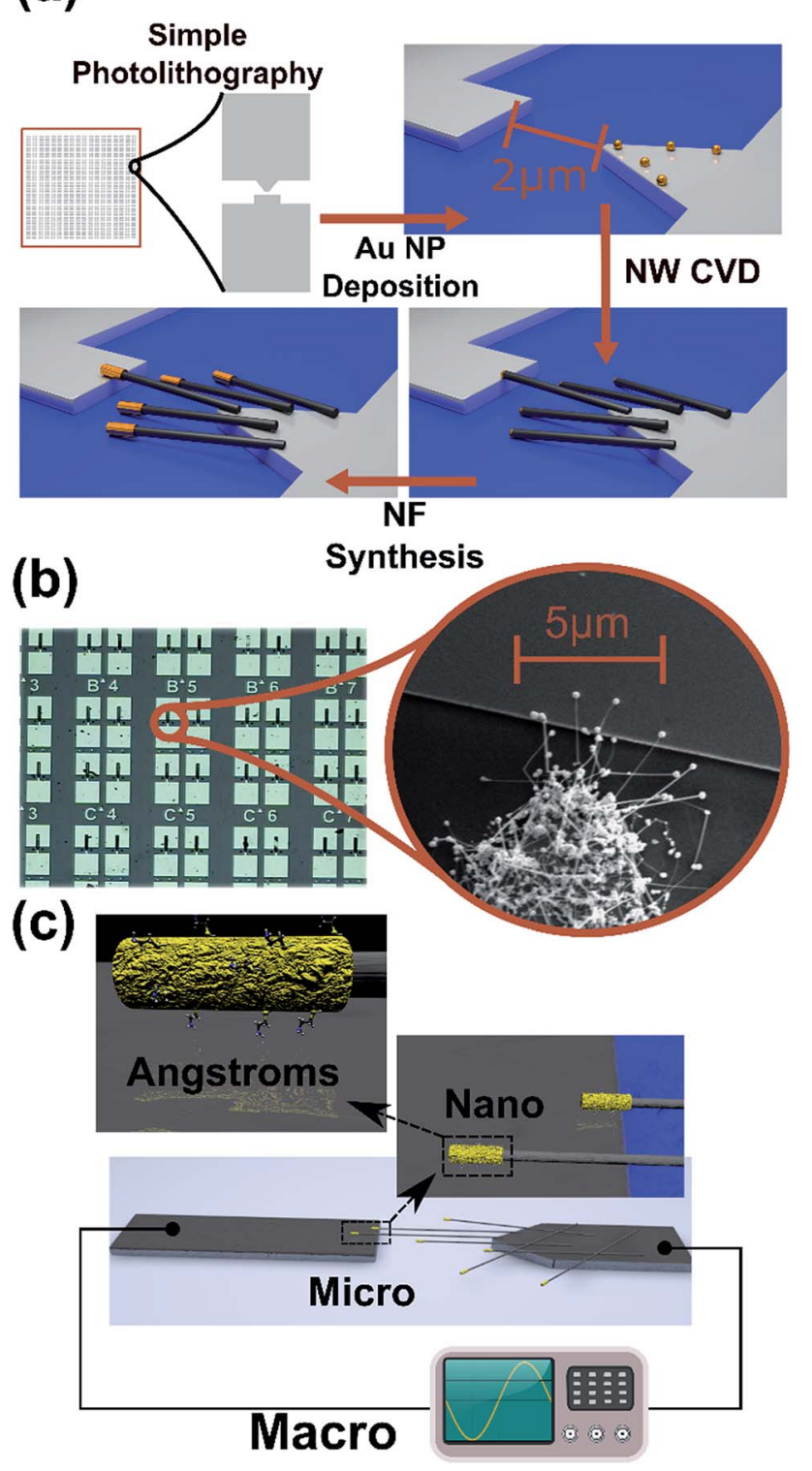

Fig. 1 NF-based nanogap electronic junction. (a) Summary of the fabrication process: first, fabrication of micro-scale metal electrodes using simple photolithography, followed by selective adsorption of gold nanoparticles on one of the electrodes that will act as catalysts for the CVD growth of the NWs. Then, insertion of the sample into a CVD system, where SiGe NWs are grown, to eventually lay horizontally to form the nanojunction. Lastly, synthesis of the NFs. (b) Optical image of a NF junction array, where the black lines correspond to locations where NFs were selectively grown. Inset: scanning electron microscope image of the nanojunction, where several NFs are shown bridging the gap between the two metal electrodes. (c) The NF junction facilitates the bridging from the nanoscale and sub-nanoscale to the macro scale.

during the NF synthesis results in collapse of the high aspect ratio HNS onto the device substrate because of capillary forces, increasing the probability of nanojunction formation.

Notably, this procedure requires simple and crude lithographic techniques and wet chemical methods, while no electrical or mechanical forces have been applied in order to align 
the NFs, unlike other alignment methods. ${ }^{23-25}$ In addition, the resulting nanojunction device is compact, requires no feedback system and is stable under ambient conditions, which makes it suitable for integration in complex measurement systems. The junction by itself is stable in various chemical and biological conditions, thus enabling measurements at variant conditions.

The inset of Fig. 1b presents an example (SEM image) of a NF-based junction device. Here, several NFs are shown bridging a micrometric gap between two metal electrodes, thus allowing current to flow when a voltage is applied across the contacts. The size of the established nanojunction depends on the contact area between the NF tip and the counter electrode, whereas in the investigated devices described here, the junction is typically $\sim 60 \mathrm{~nm}$ in length. The junction size can be altered as the NF dimensions can be controlled. ${ }^{18}$ An optical microscope image of the fabricated junctions is shown in Fig. 1b. The black lines identify the selective area where the NF is grown. Current can only flow through the NF and not through the substrate due to the $1[\mu \mathrm{m}] \mathrm{SiO}_{2}$ insulating layer on top of the substrate. Note that typically several junctions are formed. Therefore, the measurements consist of multiple nanogap junctions per device. The NF density can be varied for attaining many- or fewnanogap junction devices and by that control the device sensitivity.

Ti metal contacts bridged by the NF HNS were deposited for compatibility with the CVD process and subsequent chemical processing. These contacts were used as they form a good ohmic contact with the SiGe NF. At the tip of the NF a metalsemiconductor junction is created between the semiconducting NW and the gold nanoshell, forming a schottky junction. The NF length is determined by the duration of growth in the CVD system and can be adjusted to match or exceed the size of the gap between electrodes. Over-growth of the NWs used for NF synthesis offers additional flexibility in the fabrication process. NF HNS length can be tuned such that the contact is formed at the counter electrode either with the gold shell at the tip only, or by the semiconducting NW as well. The electronic behavior in the two cases is clearly different as shown in Fig. 2. When the contact between the counter electrode and the NF is limited to the gold shell at tip, a diode-like behavior is obtained and the nature of the junction is that of a Schottky junction. In contrast, when the semiconducting NW bridges both sides of the junction, an ohmic contact is formed on both sides and the behavior is more symmetric. These results are inline with previously performed measurements on similar Schottky junctions on Ge NWs. ${ }^{26}$ Moreover, these results demonstrate the significant influence of the gold nanoshell cap on the electronic behavior of the hybrid nanostructure, which is an important feature when introducing molecules to the system at the interface of the NF shell and counter electrode.

The electronic response of the NF junction resulting from adsorption of molecules was studied on junctions of the second type, i.e., junctions where both the gold cap and the semiconducting NW are touching the counter electrode (Fig. 2b). This was achieved by performing a sequence of three consecutive experiments for each device in an inert environment. The measurements were performed at three stages: the first measurement was taken before any chemical treatment, the second after 20 microliters of absolute ethanol was dripped onto the sample and allowed to dry, and the third measurement

\section{(a)}
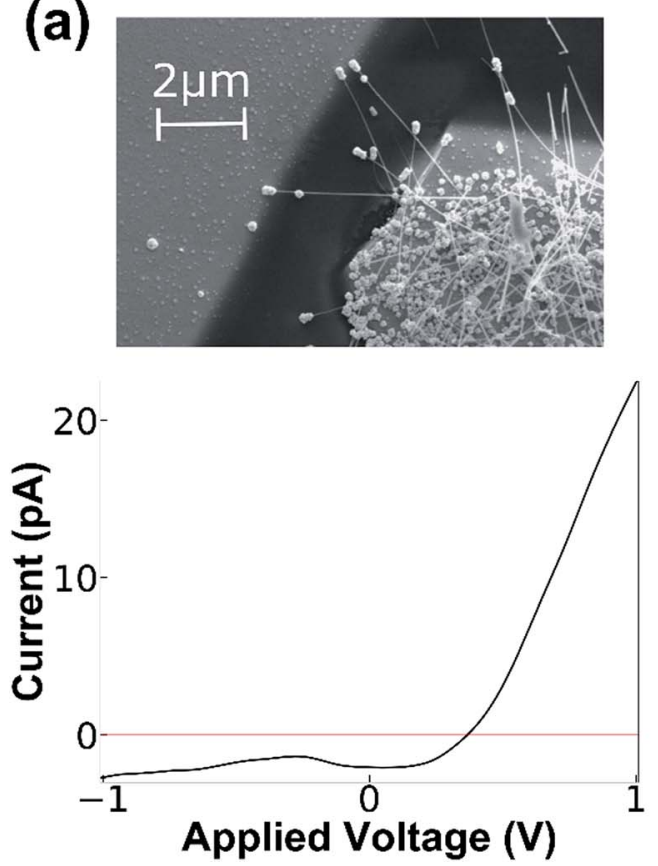

(b)
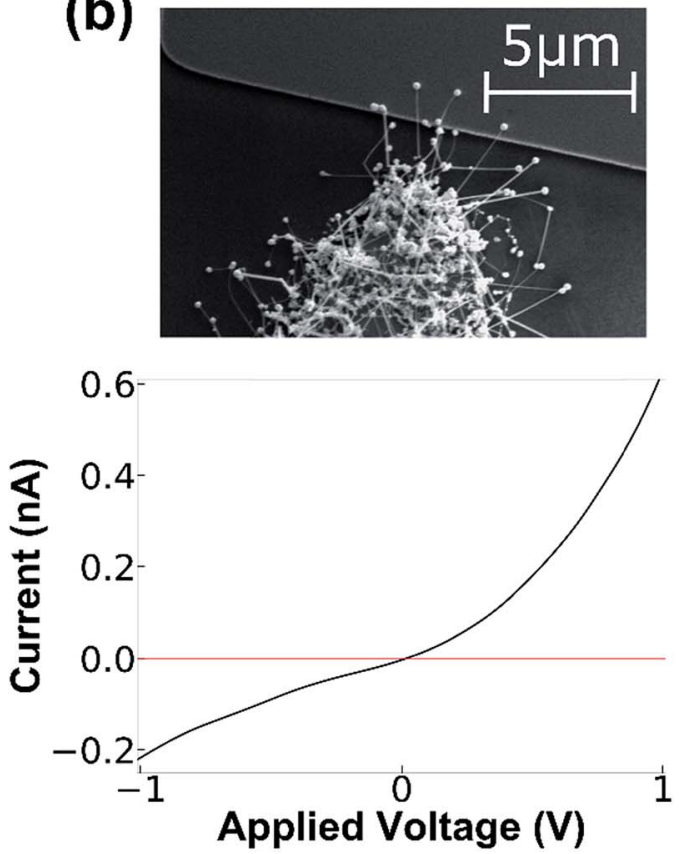

Fig. 2 Current vs. voltage measurements of NF junctions. (a) A junction measurement where only the gold nanoshell of the NF bridges the junction. This results in a rectifying behavior due to the Schottky junction between the gold and the semiconductor. (b) Both NW facet and tip contact is achieved when the NF is allowed to grow in such a way that not only the gold nanoshell touches the counter electrode but also the NW facets bridge the junction. 
was performed after dripping 20 microliters of $5 \mathrm{mM}$ ethanol solution of cysteamine.

This allowed us to identify conductance changes that might arise from the introduction of solvent apart from those related to molecular adsorption at the junction, possibly arising from mechanical changes induced, for example, by capillary forces that the solvent treatment may cause. This procedure allowed us to exclude the contribution of such effects demonstrated by two distinct examples of typical measurements in Fig. 3. In both cases, only minor differences are present between the measurement before and after ethanol treatment, however dripping the cysteamine solution resulted in a large decrease in conductance. By inspecting the numerical derivative $(\mathrm{d} I / \mathrm{d} V)$ of the first example (Fig. 3a and b) one notices a big energy gap. When molecules are captured a nanogap is formed changing the conductivity. In (Fig. $3 \mathrm{c}$ and d), the numerical derivative (dI/ $\mathrm{d} V$ ) shows that the conductance was reduced, but with no clear energy gap. These results demonstrate two cases: (1) molecules intercalate at the interface between the NF and the Ti electrode resulting in electron transport through the adsorbed molecules (see Fig. 3a and b), and (2), a change in conductance due to the
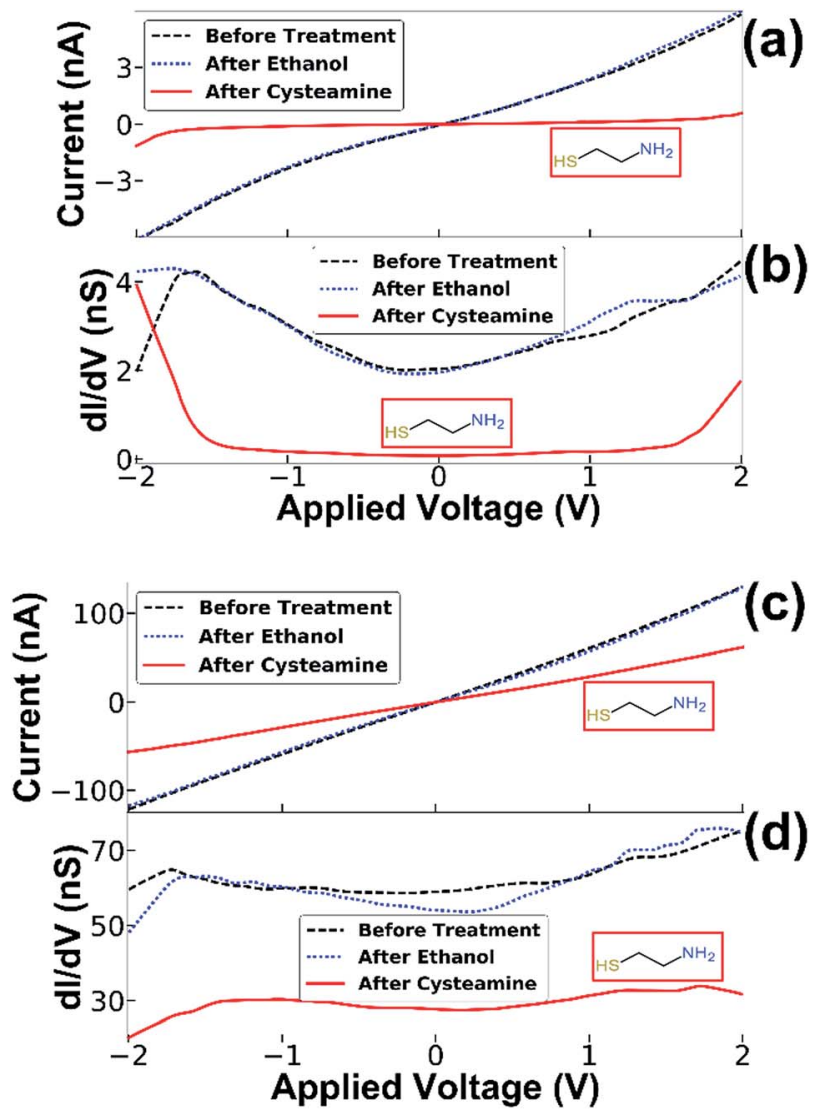

Fig. 3 Transport measurements of NF junctions. (a) Current vs. voltage characteristic and (b) its numerical derivative. The formation of an energy gap after the introduction of cysteamine molecules indicates that the electron transport occurs through the molecules. (c and d) Here, the presence of the cysteamine molecules caused a decrease in the conductance, with no energy gap. This indicates that the molecules only affect the conductance of the junction through their adsorption on the NF surface. presence of molecules on the surface of the semiconducting NW, which has altered the band bending and thus changed the electron density ${ }^{27,28}$ (see Fig. $3 \mathrm{c}$ and d). The interplay and control of the two transport mechanisms, through the SC NW surface in contact with the electrode and directly through the NF gold cap is under further study and optimization. The adsorption of molecules was further verified by XPS measurement and gold nanoparticle adsorption experiments (see ESI $\dagger$ ). All experiments were repeated several times with several batches of NF nanogap devices to ensure the generality of the results. It is important to note that a small change in the conductance after dripping only the solvent (ethanol) was observed, however, in these cases the change was random and of a smaller magnitude; i.e. in some cases the conductance was improved while in others it was reduced (with an almost equal distribution between the cases see ESI for more information $\dagger$ ). When the cysteamine molecule was introduced to the junction, the conductance was reduced in $70 \%$ of the cases. Therefore, we expect that the solvent mechanically changed the position of the NF, while the molecules actually changed the electronic properties of the junction. The generality of the NF-based nanogap devices was further demonstrated by introducing quantum dots (QDs) into the device. This fourth stage was added to the transport measurement sequence, i.e. on the same device, QD were introduced after molecules adsorption. Typical results are shown in Fig. 4, where, similar to the previous cases, introduction of molecules resulted in the characteristics energy gap shown by the numerical derivative $(\mathrm{d} I / \mathrm{d} V)$. Furthermore, the followed introduction of $2.5 \mathrm{~nm}$ CdSe QDs resulted in clear changes of the numerical derivative features, hence the energy gap was reduced and shifted, and new peaks are clearly observed at higher bias voltages (Fig. 4b). We attribute these features to tunnelling through the QD, either between the Ti contact and the gold tip or the semiconducting NW and Ti contact, so that the peaks in the $(\mathrm{d} I / \mathrm{d} V)$ curve correspond roughly to the energy

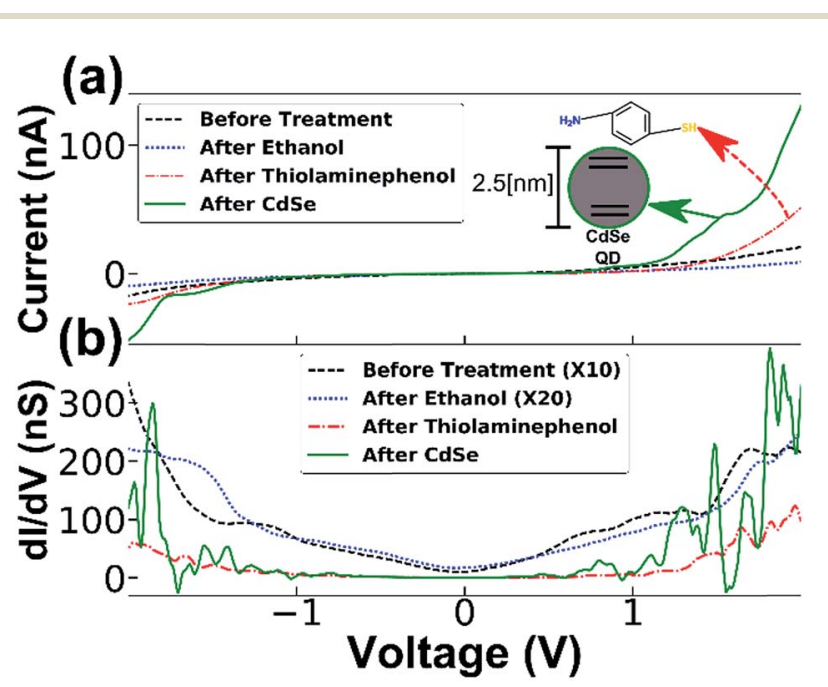

Fig. $4 \quad I-V$ curve of a NF junction device (a) and its numerical derivative (b). Only after the introduction of molecules into the system was a band gap formed, and after the insertion of CdSe QD new peaks appeared, corresponding to the QD energy levels. 
levels of the QD, considering that our measurements are performed under ambient conditions and not under UHV. ${ }^{29}$ The 2$2.2 \mathrm{~V}$ gap at around zero current is in agreement with the CdSe band gap, though this band gap is smaller than the measured photoluminescence gap of $2.35-2.45 \mathrm{eV}$ (see ESI $\dagger$ ). ${ }^{30}$ This difference is ascribed to the existence of multiple conduction channels available in the NF junction structure. We also note the appearance of negative differential conductance at the regions $\pm 1.5 \mathrm{~V}$, which supports a current flowing in parallel routes. ${ }^{29,31}$

Similar experiments were performed by applying the chemical treatment to NWs instead of NF-devices, namely, by repeating exactly the same fabrication procedure but excluding the step where NWs are processed to NF. These experiments with conventional NWs showed no formation of an energy gap. This result further supports the role of the gold nanoshell cap which is unique to the NF architecture and its key role in the trapping of molecules and QDs between the NF and the Ti contact due to the strong covalent bonds between the thiol linkers and the gold tip and its unique geometrical arrangement.

\section{Methods}

\section{Electrodes preparation}

Si substrates with $1 \mu \mathrm{m} \mathrm{SiO}_{2}$ layer (University Wafer@) were baked at $120{ }^{\circ} \mathrm{C}$ for $5 \mathrm{~min}$ to dry them. Then, they were spincoated with LOR5b for $3 \mathrm{~s}$ at $600 \mathrm{rpm}$, and then for $30 \mathrm{~s}$ at $4000 \mathrm{rpm}$. Afterwards, the samples were baked for $5 \mathrm{~min}$ at $200{ }^{\circ} \mathrm{C}$. The samples were then spin-coated with $\mathrm{nL}$ of 2020 photoresist for $2 \mathrm{~s}$ at $600 \mathrm{rpm}$ and then $40 \mathrm{~s}$ at $4000 \mathrm{rpm}$, followed by a bake for $75 \mathrm{~s}$ at $110{ }^{\circ} \mathrm{C}$. The samples were exposed to UV light using a SUSS $\odot$ MA06 mask aligner for $1.6 \mathrm{~s}$, followed by post-bake of $75 \mathrm{~s}$ at $110{ }^{\circ} \mathrm{C}$. Then, the samples were placed in an AZ-726 for $30 \mathrm{~s}$ for development. Afterwards, Ti was evaporated at $-5{ }^{\circ} \mathrm{C}$ at $1 \AA \mathrm{s}^{-1}$ followed by lift-off using $N$-methyl-2pyrrolidone (NMP) warmed to $80{ }^{\circ} \mathrm{C}, 30 \mathrm{~s}$ sonication, and rinse with isopropanol and water.

\section{Gold nanoparticle selective adsorption}

The substrates with the prefabricated contacts were baked at $120^{\circ} \mathrm{C}$ for $5 \mathrm{~min}$ to dry them. After drying, they were spin-coated with $\mathrm{nL}$ of 2020 photoresist for $2 \mathrm{~s}$ at $600 \mathrm{rpm}$ and then $40 \mathrm{~s}$ at $4000 \mathrm{rpm}$, followed by baking for $75 \mathrm{~s}$ at $110{ }^{\circ} \mathrm{C}$. Gold nanoparticles $(30 \mathrm{~nm})$ functionalized covalently with phosphonic acid (Nanopartz $\left.{ }^{\mathrm{TM}}\right)$, were dripped onto the samples $(\sim 20 \mu \mathrm{L}$, until full coverage) and left on for $30 \mathrm{~min}$. Then, the samples were washed gently in water and dried, followed by $5 \mathrm{~min}$ in acetone ("lift off"). Lastly, the sample was rinsed gently again in water and dried.

\section{Synthesis of silicon-germanium (Si-Ge) alloy nanowire (NW)}

The substrates patterned with Au NPs at specific locations were transferred to a custom-built CVD system, where SiGe NWs with an alloy composition of $1: 18$ (Si : Ge) were obtained by flowing $0.5 \mathrm{SCCM}$ of $\mathrm{SiH}_{4}$ and $90 \mathrm{SCCM}$ of $\mathrm{GeH}_{4}\left(10 \%\right.$ in $\left.\mathrm{H}_{2}\right)$ for $5 \mathrm{~min}$ at $247^{\circ} \mathrm{C}$ and 45 Torr.

\section{SiGe nanofloret (NF) synthesis}

SiGe alloy NWs that were prepared by CVD on the patterned substrates were stored in an ambient atmosphere for $48 \mathrm{~h}$ to form a native oxide layer. Then, Au-NFs were prepared by immersing the substrates with SiGe NWs in an EtOH solution (99\%, ACROS Organics) containing $1 \mathrm{mM} \mathrm{AuCl} 3$ (99\%, ACROS Organics) and 30 $\mu \mathrm{L}$ per $\mathrm{mL}$ DI water for $3 \mathrm{~min}$ at room temperature, followed by: cleaning in anhydrous ETOH twice, DI water, ETOH again, and drying under a gentle stream of $\mathrm{N}_{2}$. Samples were placed under vacuum for $3 \mathrm{~h}$ to remove moisture residues.

\section{Electron transport measurements}

The prepared samples with the NF junction were bonded using a wire bonder. All electronic measurements were performed inside a sealed chamber (Faraday cage) under constant nitrogen flow. The device was connected via BNC feedthroughs to a Keithley@ 6487 Picoammeter/Voltage Source. The voltage sweep was performed in such a way that the current under each voltage was measured 5 times and then averaged. This method results in a noise reduction of the signal.

\section{Molecules and QD solutions perpetration}

$5 \mathrm{mM}$ ethanol solution of cysteamine (Sigma Aldrich $\odot$ ) and $5 \mathrm{mM}$ ethanol solution of thiolaminephenol (Sigma Aldrich@) were prepared for the self-assembly procedure. The QDs that were used are Lumidot ${ }^{\mathrm{TM}}$ core-type CdSe $520[\mathrm{~nm}] 5 \mathrm{mg} \mathrm{mL}^{-1}$. The QDs solution was diluted 1 : 20 with anhydrous toluene.

\section{Conclusions}

A fast and simple method for the fabrication of nanometer-scale gap devices is presented using nanofloret hybrid nanostructures as building blocks with unique structural characteristics. The nanogap junction devices are used to enable electronic measurements by connecting molecules and QDs to a macroscopic electronic circuit. The unique hybrid metalsemiconductor structure of the modified NWs, nanoflorets, facilitates the intercalation of molecules and nanostructures onto the interfaces formed between the NF and the countermetal contact, which is exhibited in the characteristics of the electronic measurements and allows qualitative mapping of the semiconducting nanoparticle band structure at ambient conditions. This work establishes a first proof of concept for our platform. The new class of devices presented here were realized using relatively simple fabrication and operation procedures. These devices are attractive for exploiting the vast knowledge gained with platforms such as break junctions in a scalable platform. For applications, such devices are important in a broad context and in a variety disciplines including fundamental research of charge transport through molecules and nanostructures, sensing applications, and more.

\section{Acknowledgements}

Funding was made possible by Yissum Research Development Company of the Hebrew University of Jerusalem Ltd ("Yissum") 


\section{References}

1 A. Aviram and M. A. Ratner, Chem. Phys. Lett., 1974, 29, 277283.

2 M. Parodi, B. Bianco and A. Chiabrera, Cell Biophys., 1985, 7, 215-235.

3 M. A. Reed, C. Zhou, C. J. Muller, T. P. Burgin and J. M. Tour, Science, 1997, 278, 252-254.

4 C. Joachim, J. K. Gimzewski and A. Aviram, Nature, 2000, 408, 541-548.

5 F. S. Kim, G. Ren and S. A. Jenekhe, Chem. Mater., 2011, 23, 682-732.

6 H. Häkkinen, Nat. Chem., 2012, 4, 443-455.

7 N. A. Zimbovskaya, Transport Properties of Molecular Junctions, Springer, 2013, pp. 28-33.

8 X. Chen, Z. Guo, G.-M. Yang, J. Li, M.-Q. Li, J.-H. Liu and X.-J. Huang, Mater. Today, 2010, 13, 28-41.

9 F. Moresco, Phys. Rev. Lett., 2001, 86, 672-675.

10 B. Xu and N. J. Tao, Science, 2003, 301, 1221-1223.

11 R. H. M. Smit, Y. Noat, C. Untiedt, N. D. Lang, M. C. van Hemert and J. M. van Ruitenbeek, Nature, 2002, 419, 906909.

12 H. Park, A. K. L. Lim, A. P. Alivisatos, J. Park and P. L. McEuen, Appl. Phys. Lett., 1999, 75, 301-303.

13 S. V. Aradhya and L. Venkataraman, Nat. Nanotechnol., 2013, 8, 399-410.

14 A. Guttman, D. Mahalu, J. Sperling, E. Cohen-Hoshen and I. Bar-Joseph, Appl. Phys. Lett., 2011, 99, 063113.

15 B. Lam, W. Zhou, S. O. Kelley and E. H. Sargent, Nat. Commun., 2015, 6, 6940.

16 J. Tang, E. P. De Poortere, J. E. Klare, C. Nuckolls and S. J. Wind, Microelectron. Eng., 2006, 83, 1706-1709.

17 F. Prins, M. Buscema, J. S. Seldenthuis, S. Etaki, G. Buchs, M. Barkelid, V. Zwiller, Y. Gao, A. J. Houtepen,
L. D. A. Siebbeles and H. S. J. van der Zant, Nano Lett., 2012, 12, 5740-5743.

18 O. Hazut, S. Waichman, T. Subramani, D. Sarkar, S. Dash, T. Roncal-Herrero, R. Kröger and R. Yerushalmi, J. Am. Chem. Soc., 2016, 138, 4079-4086.

19 S. Fan, M. G. Chapline, N. R. Franklin, T. W. Tombler, A. M. Cassell and H. Dai, Science, 1999, 283, 512-514.

20 T. Jain, F. Westerlund, E. Johnson, K. Moth-Poulsen and T. Bjørnholm, ACS Nano, 2009, 3, 828-834.

21 T. Jain, S. Lara-Avila, Y.-V. Kervennic, K. Moth-Poulsen, K. Nørgaard, S. Kubatkin and T. Bjørnholm, ACS Nano, 2012, 6, 3861-3867.

22 M. Schvartzman, D. Tsivion, D. Mahalu, O. Raslin and E. Joselevich, Proc. Natl. Acad. Sci. U. S. A., 2013, 110, 15195-15200.

23 J. Yao, H. Yan and C. M. Lieber, Nat. Nanotechnol., 2013, 8, 329-335.

24 A. Pevzner, Y. Engel, R. Elnathan, T. Ducobni, M. Ben-Ishai, K. Reddy, N. Shpaisman, A. Tsukernik, M. Oksman and F. Patolsky, Nano Lett., 2010, 10, 1202-1208.

25 E. M. Freer, O. Grachev, X. Duan, S. Martin and D. P. Stumbo, Nat. Nanotechnol., 2010, 5, 525-530.

26 F. Léonard, A. A. Talin, B. S. Swartzentruber and S. T. Picraux, Phys. Rev. Lett., 2009, 102, 106805.

27 Y. Cui, Q. Wei, H. Park and C. M. Lieber, Science, 2001, 293, 1289-1292.

28 Z. Zhang and J. T. Yates, Chem. Rev., 2012, 112, 5520-5551. 29 S. Vortman, O. Ben-dor, S. Yochelis, Y. Amit and Y. Paltiel, J. Phys. Chem. C, 2013, 117, 22245-22249.

30 L. Jdira, P. Liljeroth, E. Stoffels, D. Vanmaekelbergh and S. Speller, Phys. Rev. B: Condens. Matter Mater. Phys., 2006, 73, 115305.

31 R. Pozner, E. Lifshitz and U. Peskin, J. Phys. Chem. Lett., 2015, 6, 1521-1528. 